\title{
Symmetries and invariants of the oscillator and envelope equations with time-dependent frequency
}

\author{
Hong Qin and Ronald C. Davidson \\ Princeton Plasma Physics Laboratory, Princeton University, Princeton, New Jersey 08543, USA
}

(Received 18 July 2005; published 22 May 2006)

\begin{abstract}
The single-particle dynamics in a time-dependent focusing field is examined. The existence of the Courant-Snyder invariant, a fundamental concept in accelerator physics, is fundamentally a result of the corresponding symmetry admitted by the harmonic oscillator equation with linear time-dependent frequency. It is demonstrated that the Lie algebra of the symmetry group for the oscillator equation with time-dependent frequency is eight dimensional, and is composed of four independent subalgebras. A detailed analysis of the admitted symmetries reveals a deeper connection between the nonlinear envelope equation and the oscillator equation. A general theorem regarding the symmetries and invariants of the envelope equation, which includes the existence of the Courant-Snyder invariant as a special case, is demonstrated. As an application to accelerator physics, the symmetries of the envelope equation enable a fast numerical algorithm for finding matched solutions without using the conventional iterative Newton's method, where the envelope equation needs to be numerically integrated once for every iteration, and the Jacobi matrix needs to be calculated for the envelope perturbation.
\end{abstract}

DOI: 10.1103/PhysRevSTAB.9.054001

PACS numbers: 52.20.Dq, 45.50.-j, 52.30.Gz

\section{INTRODUCTION}

The Courant-Snyder invariant for an oscillator equation with time-dependent frequency is an important concept in accelerator physics [1,2], and is a powerful constraint used to characterize the motion of a charged particle in alternating-gradient field configurations. For an oscillation amplitude $u(t)$ satisfying

$$
\ddot{u}+\kappa(t) u=0,
$$

where $\kappa(t)$ is the time-dependent frequency coefficient, the Courant-Snyder invariant is given by [1]

$$
I=\frac{u^{2}}{w^{2}}+(\dot{w} u-w \dot{u})^{2} .
$$

Here, $I=$ const (independent of $t$ ), and $w=w(t)$ is any solution of the envelope equation

$$
\ddot{w}+\kappa(t) w-\frac{1}{w^{3}}=0 .
$$

This classical result has been derived many times using different methods. The earliest derivation of the invariant known today was due to Ermakov [3], who obtained the invariant by eliminating $\kappa(t)$ from Eqs. (1) and (3). It was derived by Courant and Snyder in 1958 [1] using the basic techniques for solving Hill's equation. It was rediscovered by Lewis [4] using the asymptotic method developed by Kruskal [5]; Eliezer and Gray [6] demonstrated a physical interpretation of the invariant; a derivation using a linear canonical transformation was given by Leach [7]; and Lutzky rederived the result using Noether's theorem [8]. A short review of various derivation methods can be found in Ref. [9]. Other interesting extensions and applications related to the Courant-Snyder invariant can be found in
Refs. [10-12]. We note that the basic concept of the Courant-Snyder invariant may have appeared in other formats. For example, Kulsrud obtained two equations for $w$ which are equivalent to Eq. (3) [13]. The concept of an envelope function $w$ and its notation, we believe, can be attributed to a paper by Birkhoff [14], which predated the 1911 Solvay Conference, where, according to commonly accepted history, the concept of adiabatic invariant for a time-dependent harmonic oscillator was first discussed by Lorentz and Einstein [15].

In this paper, we first examine the time-dependent harmonic oscillator equation from the viewpoint of the symmetry group $G$ for Eq. (1). It is shown that the symmetry group for Eq. (1) is generated by an $8 \mathrm{D}$ Lie algebra (infinitesimal generator) $g$, which contains the 3D subalgebra $g_{b}$ that corresponds to the Courant-Snyder invariant [16]. The symmetry group analysis for a harmonic oscillator with constant frequency was first carried out by Anderson et al. [17], and Wulfman et al. [18] classified the corresponding Lie algebra and studied the properties of the Lie group. The envelope equation appears naturally as the determining equation for $g_{b}$. We then investigate the symmetry group of the envelope equation itself. It is interesting that the determining equation for the Lie algebra $g_{w}$ of the symmetry group $G_{w}$ for the envelope equation is an envelope equation itself. Utilizing this unique structure, a theorem regarding the symmetry and the invariant for envelope equations is derived. As an application to accelerator physics, the symmetries of the envelope equation enable a fast numerical algorithm for finding matched solutions without using the conventional iterative Newton's method, where the envelope equation needs to be numerically integrated once for every iteration, and the 
Jacobi matrix needs to be calculated for the envelope perturbation.

\section{SYMMETRY GROUP FOR THE OSCILLATOR EQUATION}

A symmetry group can be used to reduce the order of differential equations and to generate invariants [19,20]. Here, we search for vector fields $v$ in $(t, u)$ space

$$
v=\xi(t, u) \frac{\partial}{\partial t}+\phi(t, u) \frac{\partial}{\partial u}
$$

as infinitesimal generators (Lie algebra) $g$ for the symmetry transformation group $G$, which leaves Eq. (1) invariant. Here, $\frac{\partial}{\partial t}$ and $\frac{\partial}{\partial u}$ are the coordinate frames for the vector field in the $(t, u)$ space. The vector field $v$ will induce a vector field in $(t, u, \dot{u}, \ddot{u})$ space, i.e., the prolongation of $v$ denoted by $p r^{(2)} v$,

$$
\begin{gathered}
p r^{(2)} v=\xi \frac{\partial}{\partial t}+\phi \frac{\partial}{\partial u}+\phi^{u} \frac{\partial}{\partial \dot{u}}+\phi^{u u} \frac{\partial}{\partial \ddot{u}} \\
\phi^{u} \equiv \phi_{t}+\left(\phi_{u}-\xi_{t}\right) \dot{u}-\xi_{u} \dot{u}^{2} \\
\phi^{u u} \equiv-3 \xi_{u} \dot{u} \ddot{u}+\left(\phi_{u}-2 \xi_{t}\right) \ddot{u}-\xi_{u u} \dot{u}^{3}+\left(\phi_{u u}-2 \xi_{t u}\right) \dot{u}^{2} \\
+\left(2 \phi_{u t}-\xi_{t t}\right) \dot{u}+\phi_{t t},
\end{gathered}
$$

where the superdot () denotes $d / d t$, and $u, \dot{u}$, and $\ddot{u}$ are considered as independent variables. In Eq. (5), $\left(\frac{\partial}{\partial t}, \frac{\partial}{\partial u}, \frac{\partial}{\partial \dot{u}}, \frac{\partial}{\partial \ddot{u}}\right)$ denote the coordinate frames for vector field fields in the $(t, u, \dot{u}, \ddot{u})$ space. The determining equation for $v$ to be an infinitesimal generator for $G$ is

$$
p r^{(2)} v[\ddot{u}+\kappa(t) u]=\phi^{u u}+\kappa \phi+\xi \dot{\kappa} u=0 .
$$

Substituting the expression for $\phi^{u u}$, we obtain

$$
\begin{aligned}
-\xi_{u u} \dot{u}^{3}+ & \left(\phi_{u u}-2 \xi_{t u}\right) \dot{u}^{2}+\left(3 \kappa \xi_{u} u+2 \phi_{u t}-\xi_{t t}\right) \dot{u} \\
- & \left(\phi_{u}-2 \xi_{t}\right) \kappa u+\phi_{t t}+\kappa \phi+\dot{\kappa} \xi u=0 .
\end{aligned}
$$

Since Eq. (9) should be valid everywhere in $(t, u, \dot{u})$ space, the coefficients of $\dot{u}^{3}, \dot{u}^{2}$, and $\dot{u}$ should individually vanish, i.e.,

$$
\begin{gathered}
\xi_{u u}=0, \\
\phi_{u u}-2 \xi_{t u}=0, \\
3 \kappa \xi_{u} u+2 \phi_{u t}-\xi_{t t}=0, \\
-\kappa\left(\phi_{u}-2 \xi_{t}\right) u+\phi_{t t}+\kappa \phi+\dot{\kappa} \xi u=0 .
\end{gathered}
$$

Equations (10)-(13) can be used to find the solutions for $\xi$ and $\phi$. After some algebra, we obtain

$$
\begin{gathered}
\xi=a(t) u+b(t), \\
\phi=\dot{a}(t) u^{2}+c(t) u+d(t),
\end{gathered}
$$

where $a(t), b(t), c(t)$, and $d(t)$ satisfy

$$
\begin{gathered}
\ddot{a}+\kappa a=0, \\
\ddot{d}+\kappa d=0, \\
\dddot{b}+4 \kappa \dot{b}+2 \dot{\kappa} b=0, \\
\dot{c}-\frac{\ddot{b}}{2}=0 .
\end{gathered}
$$

Equations (16)-(19) have 8 degrees of freedom. Therefore, the Lie algebra $g$ is $8 \mathrm{D}$, which is the maximum dimension that a second-order ordinary differential equation can have for the Lie algebra of its symmetry group. The Lie algebra $g$ is composed of four subalgebras defined as

$$
\begin{gathered}
g_{a} \equiv\left\{a u \frac{\partial}{\partial t}+\dot{a} u^{2} \frac{\partial}{\partial u} \mid \ddot{a}+\kappa a=0\right\}, \\
g_{b} \equiv\left\{b \frac{\partial}{\partial t}+\frac{\dot{b}}{2} u \frac{\partial}{\partial u} \mid \dddot{b}+4 \kappa \dot{b}+2 \dot{\kappa} b=0\right\}, \\
g_{c} \equiv\left\{c_{0} \frac{\partial}{\partial u} \mid c_{0} \in R\right\}, \\
g_{d} \equiv\left\{d \frac{\partial}{\partial u} \mid \ddot{d}+\kappa d=0\right\} .
\end{gathered}
$$

In order for the above sets to be subalgebras, it is necessary that each set is closed under the Lie bracket. For $g_{a}, g_{c}$, and $g_{d}$, this can be trivially confirmed. Indeed, any pair of vector fields taken from $g_{a}, g_{c}$, or $g_{d}$ commute with each other. To prove that $g_{b}$ is a Lie subalgebra, we take $v_{1}, v_{2} \in g_{b}$ and

$$
\begin{aligned}
& v_{1}=b_{1} \frac{\partial}{\partial t}+\frac{\dot{b}_{1}}{2} u \frac{\partial}{\partial u}, \\
& v_{1}=b_{2} \frac{\partial}{\partial t}+\frac{\dot{b_{2}}}{2} u \frac{\partial}{\partial u},
\end{aligned}
$$

where $b_{1}$ and $b_{2}$ satisfy Eq. (18). The Lie bracket between $v_{1}$ and $v_{2}$ is

$$
\begin{gathered}
{\left[v_{1}, v_{2}\right]=b_{3} \frac{\partial}{\partial t}+\dot{b}_{3} \frac{u}{2} \frac{\partial}{\partial u},} \\
b_{3} \equiv\left(b_{1} \dot{b}_{2}-b_{2} \dot{b}_{1}\right) .
\end{gathered}
$$


Calculating the derivatives of $b_{3}$ and substituting them into the left-hand side of Eq. (18), we obtain

$$
\begin{gathered}
\dddot{b}_{3}+4 \kappa \dot{b}_{3}+2 \dot{\kappa} b_{3}=\left(b_{1} \dddot{b}_{2}-b_{2} \dddot{b}_{1}\right)+2\left(\dot{b}_{1} \dddot{b}_{2}-\dot{b}_{2} \dddot{b}_{1}\right) \\
+4 \kappa\left(b_{1} \ddot{b}_{2}-b_{2} \ddot{b}_{1}\right)+2 \dot{\kappa}\left(b_{1} \dot{b}_{2}-b_{2} \dot{b}_{1}\right)=0 .
\end{gathered}
$$

The final equality in Eq. (28) is verified by using the fact that both $b_{1}$ and $b_{2}$ satisfy Eq. (18). Therefore, $\left[v_{1}, v_{2}\right] \in$ $g_{b}$, and $g_{b}$ is a Lie subalgebra. Finally, the Lie algebra $g$ of the symmetry group $G$ can be decomposed as follows:

$$
g=g_{a} \oplus g_{b} \oplus g_{c} \oplus g_{d},
$$

where $\oplus$ denotes the sum between two linear subspaces. Furthermore, the subalgebras $g_{a}, g_{b}, g_{c}$, and $g_{d}$ are independent, and their dimensions are $2,3,1,2$, respectively.

According to the basic result of Noether's theorem, every infinitesimal divergence symmetry corresponds to an invariant [19]. Here, an infinitesimal divergence symmetry is defined as a vector field satisfying

$$
p r^{(2)} v(L)+L \frac{d \xi}{d s}=\frac{d B(t, u)}{d t}
$$

for some function $B(t, u)$. In Eq. (29), $L$ is the Lagrangian for Eq. (1). It can be shown that

$$
p r^{(2)} v(L)=\frac{d A}{d t}+\xi \frac{d L}{d t}
$$

for some function $A(t, u)$, from which it follows that $I=$ $B-A-L \xi$ is an invariant if $v$ is an infinitesimal divergence symmetry. It can also be demonstrated that every infinitesimal divergence symmetry belongs to the Lie algebra $g$ for the symmetry group $G$ of Eq. (1). Since we have obtained the Lie algebra $g$, to determine all of the invariants of Eq. (1), it is only necessary to verify which subspace of $g$ consists of infinitesimal divergence symmetries. It can be shown that the infinitesimal divergence symmetries are those from the 5D subalgebra $g_{b} \oplus g_{d}$ of the 8D Lie algebra $g$.

For the 2D subalgebra $g_{d}$, it is straightforward to show that the invariant is

$$
I=u \dot{d}-\dot{u} d,
$$

which is the well-known Wronskian for linear equations. For the 3D Lie algebra $g_{b}$, the invariant is found to be

$$
I=\left[\frac{\ddot{b}}{4}+\frac{\kappa}{2} b\right] u^{2}+\frac{b}{2} \dot{u}^{2}-\frac{\dot{b}}{2} u \dot{u} .
$$

We now show that this is indeed the Courant-Snyder invariant. Let

$$
b=2 w^{2},
$$

Eq. (18) becomes

$$
w \dddot{w}+3 \dot{w} \ddot{w}+4 \kappa w \dot{w}+\dot{\kappa} w^{2}=0,
$$

which is equivalent to

$$
\begin{gathered}
3 \dot{w} h+\dot{h} w=0, \\
h \equiv \ddot{w}+\kappa w-\frac{1}{w 3} .
\end{gathered}
$$

In other words,

$$
h=\frac{\varepsilon-1}{w^{3}}
$$

for an arbitrary real constant $\varepsilon$. Thus, we obtain the envelope equation

$$
\ddot{w}+\kappa w-\frac{\varepsilon}{w^{3}}=0 .
$$

Since $b$ is real, the $w$ defined in Eq. (33) is either real (for $b>0$ ) or purely imaginary (for $b<0$ ). When $w$ is purely imaginary, $h$ is purely imaginary as well, and $\varepsilon$ needs to be real. Therefore, Eq. (37) with a real $\varepsilon$ is valid for both purely imaginary and real $w$. The $\varepsilon / w^{3}$ term in Eq. (37) implies that $w$ cannot go through 0 . Therefore, $w$ is either a real function for all $t$ or a purely imaginary function for all $t$, which means that $b$ is either a positive definite function or a negative definite function. This fact was first proved by Struckmeier using a different method [21].

In terms of $w$, the infinitesimal generator is

$$
v_{b}=2 w^{2} \frac{\partial}{\partial t}+4 w \dot{w} u \frac{\partial}{\partial u},
$$

and the invariant in Eq. (32) becomes the familiar CourantSnyder invariant $[1,2]$

$$
I=\left(\dot{w}^{2}+\frac{\varepsilon}{w^{2}}\right) u^{2}+w^{2} \dot{u}^{2}-2 w \dot{w} u \dot{u} .
$$

In this sense, we can refer to the symmetry group generated by the subalgebra $g_{b}$ as Courant-Snyder symmetry. From the point of view of $w$, the Lie algebra of the CourantSnyder symmetry is 3D because $\varepsilon$ is an arbitrary constant in addition to the two arbitrary constants needed to specify a particular solution for $w$. Not surprisingly, Eq. (18) is exactly the same as that for the well-known $\beta$ function in Courant-Snyder theory $[1,2]$.

The 3D subalgebra $g_{a} \oplus g_{c}$ does not produce any invariant. The 1 degree of freedom associated with $g_{c}$ corresponds to

$$
v=c_{0} u \frac{\partial}{\partial u},
$$

which generates the symmetry group of the scaling transformation $\tilde{u}=\exp \left(c_{0} \tau\right) u$. This is obviously due to the fact that Eq. (1) is linear. The subalgebra of $g_{a}$ has 2 degrees of freedom, but it does not appear to have any appreciable importance. 


\section{SYMMETRY GROUP FOR THE ENVELOPE EQUATION}

We now apply the symmetry group analysis directly to the envelope equation (37) itself. The symmetry group $G_{w}$ for Eq. (37) should be a subgroup of the symmetry group $G$ for Eq. (1), because the special case of Eq. (37) for $\varepsilon=0$ is Eq. (1). Carrying out a similar procedure to that for deriving Eqs. (16)-(19), we obtain the Lie algebra $g_{w}$ for $G_{w}$ as

$$
v_{w}=2 w_{1}^{2} \frac{\partial}{\partial t}+4 w_{1} \dot{w}_{1} \frac{\partial}{\partial w},
$$

where $w_{1}$ satisfies the envelope equation

$$
\ddot{w}_{1}+\kappa w_{1}-\frac{\varepsilon_{1}}{w_{1}^{3}}=0,
$$

and $\varepsilon_{1}$ is an arbitrary real constant. Further analysis shows that $v_{w}$ is an infinitesimal divergence symmetry with the invariant

$$
I=\varepsilon\left(\frac{w_{1}}{w}\right)^{2}+\varepsilon_{1}\left(\frac{w}{w_{1}}\right)^{2}+\left(w \dot{w}_{1}-\dot{w} w_{1}\right)^{2} .
$$

We summarize the above result in the following theorem.

Theorem 1.-For an arbitrary function $\kappa(t)$ and $w_{1}, w_{2}$ satisfying

$$
\begin{aligned}
& \ddot{w}_{1}+\kappa w_{1}=\frac{\varepsilon_{1}}{w_{1}^{3}}, \\
& \ddot{w}_{2}+\kappa w_{2}=\frac{\varepsilon_{2}}{w_{2}^{3}},
\end{aligned}
$$

where $\varepsilon_{1}$ and $\varepsilon_{2}$ are real constants, the quantity

$$
I=\varepsilon_{1}\left(\frac{w_{2}}{w_{1}}\right)^{2}+\varepsilon_{2}\left(\frac{w_{1}}{w_{2}}\right)^{2}+\left(w_{2} \dot{w}_{1}-\dot{w}_{2} w_{1}\right)^{2}
$$

is an invariant.

This result was obtained by Lutzky in a less general form [8], and it can be verified in a straightforward manner by direct calculation. The invariant in Eq. (45) allows us to solve for the general solutions for $w_{1}$ in terms of a particular solution for $w_{2}$. Let $q=w_{1} / w_{2}$, we obtain

$$
I=\varepsilon_{1} \frac{1}{q^{2}}+\varepsilon_{2} q^{2}+\left(\frac{d q}{d \psi}\right)^{2}, \quad \psi \equiv \int \frac{1}{w_{2}^{2}} d t .
$$

Equation (46) can be solved for $q$ in terms of $\psi$ as

$$
q^{2}=\frac{I-\sqrt{I^{2}-4 \varepsilon_{1} \varepsilon_{2}} \sin \left[-2 \sqrt{\varepsilon_{2}}(\psi+C)\right]}{2 \varepsilon_{2}},
$$

or equivalently,

$$
w_{1}=w_{2}\left(\frac{I-\sqrt{I^{2}-4 \varepsilon_{1} \varepsilon_{2}} \sin \left[-2 \sqrt{\varepsilon_{2}}(\psi+C)\right]}{2 \varepsilon_{2}}\right)^{1 / 2} .
$$

Here, $I$ and $C$ are constants. Equation (48) recovers the Courant-Snyder theory, Eqs. (2) and (3), as a special case when $\varepsilon_{1}=0$, and $\varepsilon_{2}=1$. Another application of Theorem 1 and Eq. (48) is in the numerical solution of the envelope equation (3). For the special case of a periodic focusing lattice $\kappa(t)=\kappa(t+T)$, where $T=$ const is the period, it is desirable to find matched solutions to construct the $\beta$ functions [1,2]. Normally, this is done by an iterative Newton's method [22], where Eq. (3) needs to be solved numerically once for every iteration step. Using Eq. (48) for the case where $\varepsilon_{1}=\varepsilon_{2}=1$, we can develop a more efficient algorithm, where Eq. (3) needs to be numerically solved only once. First, we pick arbitrary initial conditions for $w(t=0)=w_{0}$ and $\dot{w}(t=0)=\dot{w}_{0}$ at $t=0$, and solve Eq. (3) numerically for $w$ from $t=0$ to one lattice period at $t=T$. Denote this particular solution by $w_{p}(t)$. Applying Eq. (48), the general solution for $w_{g}$ is

$$
\begin{gathered}
w_{g}=w_{p}\left(\frac{I-\sqrt{I^{2}-4} \sin [-2(\psi+C)]}{2}\right)^{1 / 2}, \\
\psi=\int_{0}^{t} \frac{1}{w_{p}^{2}} d t .
\end{gathered}
$$

By selecting the constants $I$ and $C$ such that

$$
w_{g}(0)=w_{g}(T) \quad \text { and } \quad \dot{w}_{g}(0)=\dot{w}_{g}(T),
$$

we obtain the matched solution to Eq. (3) for a periodic focusing lattice $\kappa(t)=\kappa(t+T)$. Of course, the matching conditions specified by Eq. (51) give two nonlinear algebraic equations for $I$ and $C$, which need to be solved using a root searching algorithm, such as Newton's method. However, we do not need to numerically integrate Eq. (3) in the root searching iterations, whereas for the conventional algorithm, Eq. (3) needs to be solved numerically once for every iteration step, and the Jacobi matrix needs to be calculated for the envelope perturbation.

As an example of this algorithm, we choose a typical focusing-off-defocsuing-off (FODO) lattice used in parti-

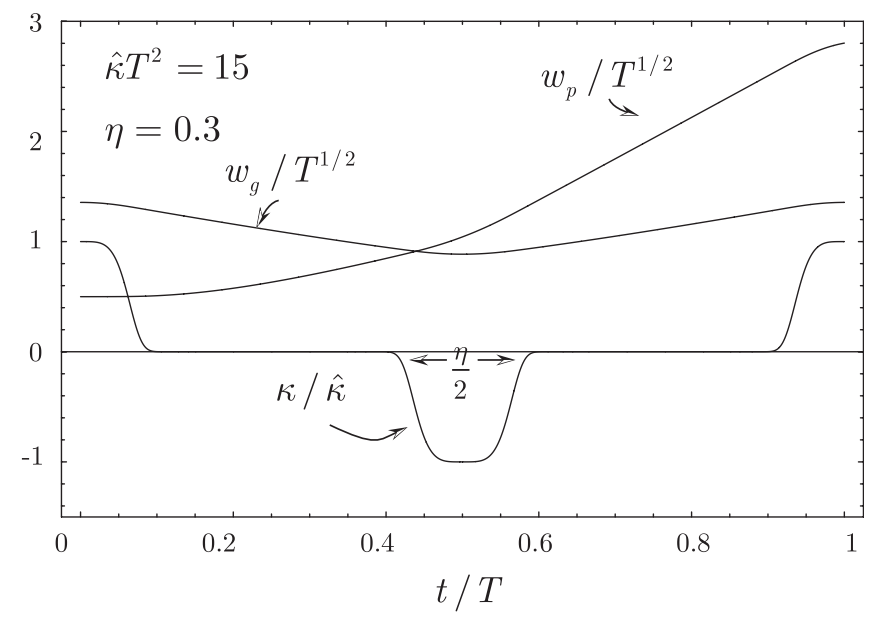

FIG. 1. The matched solution $w_{g}$ is constructed from an arbitrary special solution $w_{s}$ for a FODO focusing lattice. 
cle accelerator applications with a fringe field modeled by an Enge function [23]. The focusing lattice $\kappa(t)$ is plotted in Fig. 1. The (dimensionless) amplitude of the lattice is $\hat{\kappa} T^{2}=15$ and the filling factor is $\eta=0.3$. The particular solution $w_{p}$ is generated numerically for the arbitrarily selected initial condition $w_{p}(0)=0.5 T^{1 / 2}$ and $\dot{w}_{p}(0)=$ 0 . Obviously, $w_{p}$ is not matched. Also plotted in the figure is the matched solution $w_{g}$ constructed from $w_{p}$ using the algorithm described above. This algorithm only works for the zero space-charge $\beta$ function. It does not apply to the more general envelope equations with space charge, because the space-charge force depends on the envelope function nonlinearly and Theorem 1 does not apply.

\section{CONCLUSIONS}

We analyzed the symmetry groups of the oscillator equation and envelope equation with time-dependent frequency. It was shown that the symmetry group for the oscillator equation with time-dependent frequency is generated by an $8 \mathrm{D}$ Lie algebra, which contains a subalgebra $g_{b}$ corresponding to the Courant-Snyder invariants. The envelope equation emerged naturally as the determining equation for the subalgebra $g_{b}$. Similar analysis revealed that the symmetry group of the envelope equation itself is another envelope equation. This unique structure enables a fast numerical algorithm for finding matched solutions without using the conventional iterative Newton's method, where the envelope equation needs to be numerically integrated once for every iteration, and the Jacobi matrix needs to be calculated for the envelope perturbation.

\section{ACKNOWLEDGMENTS}

Research supported by the U.S. Department of Energy. We thank Dr. K. Takayama, Dr. T.-S Wang, Dr. P. Channell,
Dr. E. Startsev, and Dr. R. Kulsrud for many productive discussions.

[1] E. Courant and H. Snyder, Ann. Phys. (N.Y.) 3, 1 (1958).

[2] R. C. Davidson and H. Qin, Physics of Intense Charged Particle Beams in High Energy Accelerators (World Scientific, Singapore, 2001).

[3] V. Ermakov, Univ. Izv. Kiev 20, 1 (1880).

[4] H. R. Lewis, Jr., J. Math. Phys. 9, 1976 (1968).

[5] M. Kruskal, J. Math. Phys. 3, 806 (1962).

[6] C. Eliezer and A. Gray, SIAM J. Appl. Math. 30, 463 (1976).

[7] P. Leach, J. Math. Phys. 18, 1608 (1977).

[8] M. Lutzky, Phys. Lett. 68A, 3 (1978).

[9] K. Takayama, IEEE Trans. Nucl. Sci. NS-30, 2412 (1983).

[10] K. R. Symon, J. Math. Phys. 11, 1320 (1970).

[11] K. Takayama, Phys. Lett. 88A, 57 (1982).

[12] K. Takayama, Phys. Rev. A 45, 2618 (1992).

[13] R. Kulsrud, Phys. Rev. 106, 205 (1957).

[14] G. Birkhoff, Trans. Am. Math. Soc. 9, 219 (1908).

[15] H. Goldstein, Classical Mechanics (Addison-Wesley, Reading, 1980), pp. 531-540.

[16] H. Qin and R. C. Davidson, in Proceedings of the 2005 Particle Accelerator Conference (IEEE, New York, 2005).

[17] R.L. Anderson and S. M. Davison, J. Math. Analysis Applications 48, 301 (1974).

[18] C.E. Wulfman and B. G. Wybourne, J. Phys. A 9, 507 (1976).

[19] P. J. Olver, Applications of Lie Groups to Differential Equations (Springer-Verlag, New York, 1993).

[20] J. Struckmeier and C. Riedel, Phys. Rev. E 66, 066605 (2002).

[21] J. Struckmeier, J. Phys. A 38, 1257 (2005).

[22] J. Struckmeier (unpublished).

[23] M. Berz, B. Erdelyi, and K. Makino, Phys. Rev. ST Accel. Beams 3, 124001 (2000). 